dit

TRANS $\cdot \mathrm{N}^{\circ} 7 \cdot 2003$

El presente articulo aborda el tratamiento SECCIÓN - 59-70 teórico que se le ha venido dando últimamente a la recepción de la literatura traducida. Para ello nos ocuparemos del concepto de polisistema de Itamar EvenZohar, de las normas de Guideon Toury y de lo que Lawrence Venuti denomina invisibilidad del traductor. Al mismo tiempo, haremos referencia a los distintos métodos de análisis que tanto estos teóricos como otros han propuesto para el estudio de la literatura traducida.

\title{
Recepción literaria y traducción: estado de la cuestión
}

The present paper sets out the most recent theoretical treatment of translated literature and will approach Itamar EvenZohar's Polysystem theory, Guideon Toury's Norms, and what Lawrence Venuty calls The Translator's Invisibility. While referring to the diverse analytical methods proposed by these scholars we will refer to others who sought to further define the studies of translated literature.

JoRge LeIVA Rojo

Univ. Pablo de Olavide 


\section{INTRODUCCIÓN Y CONCEPTOS CLAVE}

La noción de recepción literaria, tal y como la entendemos hoy, surgió a finales de la década de los sesenta, gracias al influjo de la Escuela de Constanza, representada especialmente en las personas de Hans Robert Jauss y Wolfgang Iser (Starobinski, 1987: 214; Acosta Gómez, 1989: II8; Iglesias Santos, 1994a: 36-37). Fue el primero de ellos quien propuso un cambio de paradigma a la hora de abordar el estudio de la ciencia literaria, pues consideraba que se había agotado la validez de los paradigmas clásicohumanista, histórico-positivista y estético-formalista, tan vigentes en otros tiempos (Segers, I979: 83-84; Jauss, I987a: 59 y 69; Iglesias Santos, I994a: $39-40$ ).

La nueva noción de recepción literaria, en la que el autor permite que el lector tome parte de su experiencia (Jurt, 1998: 44), ejercerá una enorme influencia en todo el ámbito de la literatura, pero también en disciplinas adyacentes, como la sociología o la historia del arte (Holub, 1985: XI). A partir de ese momento, la teoría de la recepción literaria supondrá una revolución no solo porque el lector asumirá un papel relevante para esta (Bark, 1987: 17r; Iser, 1987a: 121 y 1987b: 149; Acosta Gómez, 1989: I7; Iglesias Santos, 1994a: 35 ; Jurt, I998: 48 y 64), sino porque también comenzará a hablarse de la literatura como medio de comunicación y fenómeno histórico que tiene una marcada función social y es un sistema de signos de estructura significativa (cf. Jauss, I987a: 67; Acosta Gómez, I989: 18-23).

El precursor de esta nueva visión de la literatura y, por ende, de la recepción literaria, es Aristóteles, quien en su obra Poética, por su inclusión de la catarsis como categoría central de la experiencia estética, puede ser considerado la manifestación más temprana de una teoría en la que el público desempeña un papel primordial. Le seguirían posteriormente los dos principales representantes de la estéti- c a del siglo XVIII: Baumgarten y Kant, este último escritor en 1790 de Critica del juicio (Holub, 1985: I3; Jauss, 1987b: 73; cf. Weinrich, I987: 200).

$¿$ Qué se entiende entonces por recepción literaria? Acosta Gómez (1989: 13) considera que puede ser definida de la siguiente forma:

El conocimiento, acogida, adopción incorporación, apropiación o crítica del hecho literario en cuanto operaciones realizadas por el lector, o como la adaptación, asimilación o incorporación de una obra en tanto que actividades llevadas a cabo por otro escritor.

Han sido muchos los estudiosos que han tratado la recepción literaria desde diversos puntos de vista. Sin embargo, nosotros nos vamos a centrar en aquellas corrientes que han tenido como punto común el estudio de la recepción literaria en relación con la traducción, haciendo especial hincapié en los conceptos clave introducidos por éstas.

\section{POLISISTEMAS}

El profesor israelí Itamar Even-Zohar enuncia su teoría del polisistema en la década de los setenta, tomando como base el pensamiento de los formalistas rusos de los años veinte y el estructuralismo de la Escuela de Praga (Even-Zohar, I979: 290; Bassnett, I998: 125; Gentzler, 1993: 109; Hermans, 1999: 106). La teoría del polisistema, si bien la concibió Even-Zohar en un principio para su aplicación al lenguaje (Hermans, I999: 106), supone una revolución en el sentido de que propone que las obras literarias no se estudien como algo aislado, sino que se les considere parte de un sistema literario (Iglesias Santos, I994b: 312; Lambert, 1998: 132; Marco, 2002: 26). Es precisamente el concepto de sistema, definido como el conjunto «of functions of the literary order which are in continual interrelationship 
with other orders»' (Tynjanov, ápud Munday, 200I: I09), la contribución más destacada de los formalistas a la obra de Even-Zohar (Shuttleworth, 1998: 176). Según la definición que da Tynjanov se deduce que la literatura forma parte del marco social, cultural, literario e histórico. En el concepto clave, el de sistema, está presente por tanto una dinámica progresiva de mutación y lucha para alcanzar una posición destacada en el canon literario.

En lo dicho anteriormente se basa EvenZohar para rechazar el modelo impuesto tradicionalmente, que se ha centrado únicamente en la denominada literatura "alta" y ha restado importancia a sistemas literarios o géneros como la literatura infantil, los libros de suspense y la literatura traducida (Even-Zohar, 1979: 292; Shuttleworth, 1998: 177). De esta última llega a decir que opera como un sistema y argumenta su decisión en dos razones (Munday, 200r: I09):

I) Porque la LM selecciona obras para traducirlas.

2) Por la influencia que otros co-sistemas ejercen en las normas y las políticas de traducción.

Las relaciones existentes entre los sistemas las enmarca Even-Zohar en un nuevo concepto, para el que acuña la denominación de polisistema y que, según señala Shuttleworth, es en realidad sinónimo de sistema. La definición que da este último del término polisistema es la siguiente:

[t]he polysystem is conceived as heterogeneous, hierarchized conglomerate (or system) of systems which interact to bring about an ongoing, dynamic process of evolution within the polysystem as a whole. ${ }^{2}$ (Shuttlerworth, I998: 176)

\footnotetext{
«de funciones del orden literario que está en continua interrelación con otros órdenes». (Las traducciones son del autor).

2 "[e]l polisistema se concibe como un conglomerado (o sistema) de sistemas, heterogéneo y jerarquizado, que interacciona para dar como resultado un proceso de evolución continuo y dinámico que tiene lugar dentro del polisistema, considerado como un todo».
}

El proceso de evolución dinámico al que se refiere Shuttleworth, señala Chesterman (I997: 37), es vital para el polisistema, pues indica que las relaciones que se establecen entre los sistemas primario -innovador-y secundario conservador - se encuentran en un continuo estado de cambio y competición. Consecuencia directa de ello es que la posición que ocupa la literatura traducida en el polisistema tampoco sea fija, de manera que puede ocupar en él una posición primaria o una posición secundaria (Even-Zohar, 1981: 2-3; I990: 46; Gentzler, 1993: II7; Iglesias Santos, I994b: 331; Hermans, 1999: 109). Si su posición es primaria, indica Even-Zohar, participa activamente en el proceso de modelado del centro del polisistema, es decir, su influencia es destacada.

Los supuestos en que la literatura traducida ocupa la posición primaria $y$, por tanto, contribuye a la elaboración de nuevos repertorios, los reduce Even-Zohar a tres (Even-Zohar, I979: 296-300; I990: 47; I997: 359; Gentzler, I993: I17; Bassnett, 1998: 127; Shuttleworth, 1998: 177-178; Hermans, I999: 109):

a) Cuando una literatura «joven» se encuentra en sus inicios y se fija inicialmente en literaturas «viejas» para adoptar modelos ya creados.

b) Cuando una literatura es «periférica» o «débil» e importa los modelos literarios de los que carece.

c) Cuando se produce un punto de inflexión desde el punto de vista de la historia de la literatura, que provoca que no se consideren suficientes los modelos establecidos, o bien cuando existe un vacio en la literatura de un determinado país. Cuando no existe ningún modelo dominante, los modelos extranjeros pueden adquirir primacía con mayor facilidad.

En el caso de que la literatura traducida ocupe una posición secundaria, se entenderá que esta representa un sistema periférico dentro del polisistema. Es esta la situación que Even-Zohar considera más frecuente. Al mismo tiempo, afirma que la posición que

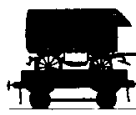

61 


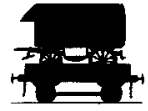

ocupe la literatura traducida en el polisistema va a estar estrechamente ligada con el resultado de la traducción, de manera que, si la traducción que se está realizando se encuadra dentro de la posición primaria, el traductor no se verá obligado a seguir los patrones de la LM y, por lo tanto, las probabilidades de que se rompan convenciones y, como consecuencia, se creen nuevos modelos en la LM serán mayores (cf. Even-Zohar, 1981: 3). Por el contrario, si la traducción ocupa una posición secundaria, la tendencia más común es que el traductor haga uso de modelos culturales preexistentes en la $\mathrm{LM}$ (Even-Zohar, I990: 5I).

Considera Theo Hermans (1985: 12) que la teoría de los polisistemas proporciona el marco adecuado para el estudio sistemático de la literatura traducida, pues opina que su sencillez facilita que pueda ser fácilmente adoptada como herramienta cognitiva, al tiempo que es lo suficientemente flexible e inclusiva como para poder adaptarse a los diferentes casos y situaciones. Shuttleworth (1998: I79) dice de ella que, si bien no es una teoría completa, constituye el punto de partida para investigaciones posteriores, ya sean teóricas o descriptivas.

Gentzler (I993: I20-I2I, I24), por su parte, destaca que sea capaz de integrar el estudio de la literatura con el estudio de las fuerzas económicas y sociales de la historia, al tiempo que reconoce su aportación no solo a la teoría de la traducción literaria, sino también a la teoría de la literatura. Sin embargo, este mismo autor considera que la parte más controvertida de esta teoría es la tendencia a la generalización y al establecimiento de universales sin contar con numerosas pruebas, lo que le lleva a formular principios en ocasiones contradictorios. Susan Bassnett (1998: 127) opina que el planteamiento de Even-Zohar conlleva la dificultad de establecer los criterios para determinar si una literatura es periférica o débil, extremo en el que coincide Hermans (I999: II7-II9), quien, a su vez, tilda la teoría del polisistema de imprecisa. Lefevere (ápud Hermans, 1999: 125), por su parte, rechaza dicha teoría por considerar que los sistemas a los que alude Even-Zohar no existen, al tiempo que considera que la oposición entre lo primario y lo secundario es superflua.

\section{NORMAS}

El término descriptive translation studies o translation studies, propuesto por Holmes en I972 (Holmes, 2000: 175-176) para definir la preocupación académica, teórica, empírica o aplicada referida a cualquier aspecto de la traducción, hizo posible que se llegara al consenso en lo referente a la denominación de la disciplina (Gentzler, 1993: 92-93; Hermans, 1999: 8).

$\mathrm{El}$ análisis, en el marco de los estudios de traducción, de las normas de traducción, entendidas como la regularidad en comportamiento y el mecanismo subyacente causante de dicha regularidad, ha sido abordado, entre otros, por Levy', Popovič y Mukařovský (Hermans, I999: 73-80). Sin embargo, es Guideon Toury el máximo exponente de esta corriente, que supone una continuación del modelo trazado por Even-Zohar, ya que considera que la posición que ocupa una traducción en los sistemas social y literario de la cultura meta determina el tipo de estrategia traductiva que se habrá de emplear (Toury, 1980: 35). Al mismo tiempo, se plantea la necesidad de establecer un enfoque que, basado en el TM, pueda servir de base empírica al estudio de la literatura traducida (Toury, 1980: 36; 1981: 9; Gentzler, 1993: 127; Baker, 1998: I63). Por otra parte, considera que la mayoría de las corrientes existentes han formulado teorías de traducción orientadas al To, que él considera inadecuadas, puesto que lo idóneo, según Toury (I980: 40; 1985: I9; Hermans, 1985: 13), es que se centren en el TM, para lo que arguye que el traductor actúa de acuerdo con un interés primordial por la cultura meta, $y$ no por la de origen. En lo que a la metodología se 
refiere, Toury propone los siguientes pasos a la hora de abordar el estudio de las traducciones (Toury, I980: II7-II8):

I) Situar el texto dentro de la cultura meta y comprobar su relevancia y aceptación.

2) Comparar fragmentos del To y el TM e identificar las relaciones que se establecen entre ellos y que son generales y subyacentes al concepto de traducción. Opina Munday (200r: 123) que este aspecto es el más controvertido del pensamiento de Toury, puesto que considera este último que los fragmentos que se han de analizar los debe determinar la teoría de la traducción. Es precisamente en este punto donde surge el problema, ya que la teoría de la traducción, indica Munday, está lejos de llegar al consenso. A este respecto señala Holmes la conveniencia de elaborar una lista de aspectos que se deberían examinar. Por lo demás, esta fase de estudio viene a suponer la existencia de una traducción adecuada que sea capaz de satisfacer cualquier necesidad lingüística o cultural, supuesto que posteriormente se encargaría de rechazar el propio Toury (Gentzler, I993: I28).

3) Extraer conclusiones y establecer líneas generales que faciliten la toma de decisiones en futuras traducciones.

Como hemos apuntado anteriormente, considera Toury que la traducción está regida por normas, $y$ son precisamente dichas normas las que determinan el tipo de equivalencia aplicable a las traducciones. Por norma se entiende, en definitiva, «the translation of general values or ideas shared by a certain community - as to what is right and wrong, adequate and inadequate - into specific performance-instructions appropriate for and applicable to particular situations” ${ }^{3}$ (Toury, 1980: 5 I). Es decir, las

3 «la translación de valores o ideas generales compartidos por una comunidad determinada - en cuanto a lo que es acertado y erróneo, adecuado e inadecuado- $-a$ instrucciones de actuación específicas, adecuadas y aplicables a situaciones concretas". normas a las que se refiere Toury no son sino restricciones socioculturales específicas para una cultura, para una sociedad y para un tiempo, restricciones que adquieren los individuos a través del proceso de educación y socialización (cf. Toury, I980: 52).

The aim of Toury's case studies is to distinguish trends of translation behaviour, to make generalizations regarding the decisionmaking processes of the translator and then to 'reconstruct' the norms that have been in operation in the translation and make hypotheses that can be tested by future descriptive studies.4 (Munday, 200I: Ir3)

Con estas palabras define a la perfección Munday qué propósito lleva a Toury a enunciar su teoría sobre el concepto de norma. De aquí se deduce que Toury defiende que a través del producto - la traducción- se pueden identificar los procesos que han intervenido - las normas-. Las normas que han prevalecido en la traducción de un texto concreto se pueden reconstruir a través de dos fuentes (Toury, 1980: 57; 2000: 207; Hermans, 1999: 85):

a) Textuales: el análisis de los textos en sí mostrará las «regularidades de comportamiento", es decir, los procesos adoptados por el traductor $y$, por lo tanto, las normas que han participado.

b) Extratextuales: se trata de las teorías prescriptivas de la traducción, así como de las declaraciones referentes a las normas efectuadas por traductores, editores, críticos, lectores o cualquier otro participante en el proceso de la traducción. Sin embargo, advierte Toury de que dichas declaraciones pueden estar incompletas o sesgadas, debido a la subjetividad, ingenuidad

4 «El propósito de los estudios de Toury es distinguir las tendencias del comportamiento traductivo, establecer lineas generales referentes a los procesos de toma de decisiones del traductor y, a partir de ahí, ureconstruir" las normas que han intervenido en la traducción y emitir hipótesis que puedan ser analizadas en estudios descriptivos venideros».

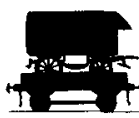




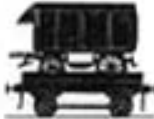

o falta de conocimiento suficiente por parte de quienes las emitieron.

Por otra parte, distingue Toury tres tipos de normas distintas. En primer lugar se sitúa la norma inicial, mientras que en un nivel inferior se encuentran, de una parte, las normas preliminares $y$, de otra, las normas operacionales (Toury, 1980: 53-55 y $115-116$; 1981; 23-24; 1985: 24; 2000: 200-204; Gentzler, 1993: 130-131; Chesterman, 1997: 63-64; Baker, 1998: 164; Hermans, 1999: 75-76). 5

a) La norma inicial hace referencia a la decisión general adoptada por el traductor, de manera que el traductor puede optar por ceñirse a las normas del To, o bien inclinarse por las normas del TM o la cultura meta. En el primero de los casos, habla Toury de traducción adecua$d a$, mientras que a la traducción resultante de aplicar las segundas normas la considera aceptable. Sin embargo, ambos extremos suelen combinarse, ya que no existe traducción que pueda ser considerada totalmente adecuada o totalmente aceptable.

3 Sin embargo, no esta la única clasificación de normas existente. De esta forma, Chesterman (1997: 64-70; Hermans, 1999: 77-78; Baker, 1998: 165; Munday, 200r: n8ug) distingue entre:

a) Normas de expectativa o de producto, que son las que están determinadas por lo que lo lectores esperan del TM. En ellas ejercen una gran influencia la tradición traslativa de la cultura meta y los textos paralelos existentes en la LM. Así mismo, desempeñan un papel importante los factores ideológicos y comerciales $y$ las relaciones entre las culturas en cuestión.

b) Normas profesionales, que regulan el proceso de la traducción y dependen de las primeras. Chesterman las divide en tres tipos: norma de responsabilidad, referente al sentido de lealtad del traductor a la obra original y al autor; norma de comunicación, basada en el principio de optimizar la comunicación entre las partes involucradas; y norma de relación, en la que subyace el concepto de equivalencia que debe primar entre el To y el TM.

Nord (Hermans, 1999: 79), por su parte, habla de:

a) Normas constitutivas, que determinan lo que una cultura determinada denomina traducción como término opuesto a los conceptos de adaptación y verrión.

b) Normas reglamentarias, referidas a las formas generalmente aceptadas de solventar los problemas de traducción que puedan surgir. b) En las normas preliminares se integran las políticas de traducción, que hacen referencia a los factores que determinan la selección de textos para ser traducidos en una cultura, tiempo o lengua en concreto. Por otra parte, $\mathrm{l}_{\mathrm{a}}$ directness de la traducción hace referencia a si ha mediado otro texto entre el To y el TM. Dentro de este ámbito, sugiere Toury el estudio de la tolerancia de la cultura meta hacia esta práctica, qué lenguas intervienen y si dicha práctica se camufla o no.

c) Las normas operacionales, que describen la presentación y la cuestión lingǘística del TM, integran por un lado a las normas matriciales, encargadas de hacer referencia a la manera en que se distribuye en el TM el material lingüistico. Los fenómenos que se pueden estudiar son, por poner un ejemplo, la omisión o cambio de emplazamiento de fragmentos, la segmentación textual y la adición de fragmentos o de notas al pie. Por otra parte, también se incluyen aquí las normas lingüístico-textuales, que rigen la selección del material lingüístico existente en el TM: rasgos léxicos, fraseológicos y estilísticos.

En definitiva, los estudios descriptivos de traducción tienen como objetivo la reconstrucción de las normas que han intervenido en el proceso de traducción (Toury, 1985: 37; Lefevere, 1995: 7). Sin embargo, considera el propio Toury que el de norma es un concepto gradual, ya que la actuación del traductor no puede esperarse que sea totalmente sistemática (Toury, 2000: 205).

Gentzler (1993:133-134) considera que las principales aportaciones de la teoría de Toury a los estudios descriptivos de traducción son las cuatro siguientes:

a) El abandono de las nociones clásicas de correspondencia.

b) La involucración de las corrientes literarias del sistema cultural de la lengua meta en la producción de textos traducidos.

c) La desestabilización de la noción de mensaje original con una identidad fija. 
d) La integración del texto original y del texto traducido en la red semiótica de sistemas culturales que se relacionan entre sí.

Vanderschelden (2000: 273), por su parte, considera que la obra de Toury ha supuesto una revolución en la forma de abordar el estudio de calidad en la traducción, mientras que Baker advierte de su gran influencia durante la década de los ochenta y los noventa, hasta el punto de afirmar que esta teoría «has supported the most active research programme in translation studies to date ${ }^{6}$ (Baker, I998: I63). Sin embargo, debe decirse que no todo fueron elogios para el pensamiento de Toury. Así, considera Munday (200I: II7) que las mismas críticas que vertió Gentzler a la teoría de los polisistemas pueden aplicarse a la aportación de Toury, esto es, el deseo de generalizar a través del estudio de los casos y la aplicación de unas normas abstractas a las que se le achaca estar dominadas por el subconsciente de su propio autor. Berman, por su parte, critica las normas, porque suponen una negación de la creatividad de la traducción y del traductor, mientras que Hermans cuestiona la posibilidad de poder establecer unos patrones generales aplicables a la toma de decisiones del traductor (Hermans, 1999: 154; cf. Berman, 2000: 296). Al mismo tiempo, señalan Chesterman (1997: 64) y Hermans (1999: 77) el inconveniente de oponer dos términos como adecuado y aceptable, razón por la que este último propone el empleo de orientado al TM y orientado al TO. ${ }^{7}$

«se ha convertido en el programa de investigación en estudios de traducción más activo hasta la fechas.

' La confirmación de esta idea se ve reflejada en el hecho de que fuera el mismo Toury quien, en 1995, desechara por erróneo e ineficaz el concepto de traducción adecuada que êl mismo había enunciado (Hermans, 1999: 56-57).
Considera Venuti que el concepto de norma propuesto por Toury presenta como principal carencia el hecho de que no se tenga en cuenta la importancia que ejercen las editoriales en el proceso de traducción, ya que estas en multitud de ocasiones determinan el método de traducción que se habrá de emplear, al tiempo que opina que el estudio de la traducción no puede ser simplemente descriptivo (cf. Venuti, 1985: 162; 1995a: 312).

Venuti emplea el término invisibilidad para referirse a «the translator's situation and activity in contemporary Anglo-American culture ${ }^{8}$ (Venuti, I995a: I), situación que también advierte Hale (1998: 194). La invisibilidad, que Pym (1996: 171) asegura que no es exclusiva de la cultura anglo-americana, se puede apreciar, de una parte, a través de la forma en que los traductores abordan la traducción del texto, con el objeto de conseguir un TM en el que prevalezca la ilusión de transparencia de la que habla Venuti y, de otra, a través de la lectura que se hace del texto en la cultura de llegada, lectura en la que se da prioridad a la sensación de que el TM no aparente ser una traducción, sino el To (Lambert, 1998: 13r; Munday, 200r: 146). En estas afirmaciones encuentra Baker el escaso o nulo interés por estudiar el estilo del traductor, que hasta la actualidad siempre se ha negado, ya que su labor se ha venido considerando que debe ser la de reproducir lo más exactamente posible el estilo del original (Baker, 2000: 244). Sin embargo, es difícil lograr una total ilusión de transparencia por parte del traductor; así lo ve Hermans (1996: 26), quien se pregunta si «the translator, the manual labour done, disappear[s] without textual tracen ${ }^{9} \mathrm{y}$ da como posible respuesta que

8 ala situación y la actividad del traductor en la cultura angloamericana contemporánear.

9 *el traductor, la labor manual realizada, desaparece sin dejar huella textuals.

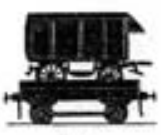

65 


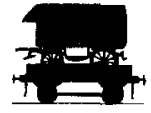

el estilo del traductor se manifieste en la voz del narrador (Hermans, 1996: 27 y 43).

Baker, quien cuestiona lo afirmado por Hermans, considera que el estilo del traductor $y$, por tanto, la visibilidad o invisibilidad de este, puede apreciarse igualmente a través de una serie de rasgos, como el hecho de que haya elegido él mismo el texto para traducir, así como el uso de una serie de estrategias, entre las que se incluyen el empleo de introducciones, epilogos y notas al pie (Baker, 2000: 245). Al mismo tiempo, deberá tenerse en cuenta el uso que hace el traductor de aspectos del lenguaje, como por ejemplo el léxico, la sintaxis o la puntuación, con respecto a otros traductores y con respecto al propio To (Baker, 2000: 248 y 255$).^{10}$

Complementarios al concepto de invisibilidad son los dos tipos de estrategias de traducción que distingue Venuti: la domesticación y la extranjerización.

La domesticación es la tendencia que Lawrence Venuti (I995b: Io y 17) considera que prevalece en la cultura traductológica angloamericana $^{\text {II }}$. Este fenómeno, que consiste básicamente en «an ethnocentric reduction of the foreign text to target-language cultural values ${ }^{12}$ (Venuti, 1995a: 20), supone traducir tomando como modelo un estilo transparente, fluido e invisible, de manera que se pueda reducir al máximo la presencia de rasgos extranjeros en el TM (cf. Venuti, 1992: 4; I995a: 20; I995c: 27; Schäffner, r995: 4; Chesterman, I997: 169).

to En relación con este aspecto, véanse las doce tendencias que, según Berman (2000: 288-297), están presentes, por norma general, en los textos traducidos.

Anthony Pym (1996: I70) opina que la domesticación es también la tendencia dominante en otros países, como Brasil, España y Francia, extremo que confirma Vanderschelden (2000: 273 y 287) en referencia a este último país. Basil Hatim, por su parte, habla de la domesticación como la estrategia de traducción más habitual en los países occidentales (Hatim, 1999: 204).

12 «una reducción etnocéntrica del texto extranjero a los valores culturales de la lengua de llegada».
Hatim (I999: 2I4), por su parte, identifica la domesticación con la traducción libre, pues supone «negotiating the discoursal, generic and textual designs of the source text in terms of target language norms and conventions $m^{13}$.

En el mismo sentido se expresa Venuti al afirmar que la domesticación supone que el lector pueda reconocer su propia cultura en otras culturas, lo que viene a revertir en un dominio del imperialismo cultural del que salen más beneficiadas las editoriales y más perjudicados los traductores (Venuti, 1992: 5; I998b: 124). En este concepto se encuentra una clara alusión al modelo de traducción que Schleiermacher define como aquel que «leaves the reader in peace, as much as possible, and moves the author towards him ${ }^{14}$ (Schleiermacher, ápud Munday, 200I: 146). Al mismo tiempo, es posible apreciar claras conexiones con la estrategia de naturalización que ya enunciara y criticara Berman en 1985 (Venuti, I995b: 22), puesto que considera que el propósito ético del acto de traducción es recibir lo extranjero como tal.

Por su parte, Hatim relaciona la domesticación con la traducción encubierta de la que hablan Gentzler (1993: 138), Hermans (I999: 65), Lambert (1998: 130) y House (1998: 214), que es el tipo de traducción no marcada como tal y que, por lo tanto, pretende introducirse en la cultura meta como si de un texto origen se tratara. Finalmente, señala Fawcett (ápud Munday, 200r: 154) que, debido a la influencia ejercida por editores y correctores, el producto final es, en ocasiones, una traducción domesticada.

La segunda estrategia a la que hace referencia Venuti, la extranjerización, «entails choosing a foreign text and developing a translation

\footnotetext{
13 «negociar el diseño discursivo, genérico y textual del texto origen en términos de las normas y convenciones de la lengua meta».

I4 «molesta al lector lo menos posible y aproxima el autor al lector".
} 
method along lines which are excluded by dominant cultural values in the target language ${ }^{15}$ (Venuti, I998a: 242), es decir, consiste en dar especial importancia a las diferencias lingüísticas y culturales del To y plasmarlas tal cual en el TM (cf. Schäffner, I995: 4). Hatim, por su parte, considera que la extranjerización supone «negotiating these values [the discoursal, generic and textual designs] in terms of source language norms and conventions ${ }^{16}$ (Hatim, I999: 214). La traducción extranjerizante, en definitiva, se relaciona con el concepto de traducción manifiesta de House, un tipo de traducción en el que no se le invita a participar al lector meta, sino que este se limita a ser mero observador (Hatim, 1999: 214). Esta opción, la más empleada en traducción literaria durante el siglo XX (Johnston, 1992: 45) y la más acertada según Schleiermacher (Lefevere y Bassnett, I998: 8), la define este último como la estrategia de traducción donde «the translator leaves the writer alone as much as possible and moves the reader toward the writer" ${ }^{17}$ (Schleiermacher, ápud Bernofsky, 1997: 176).

Igualmente considera Venuti que la traducción extranjerizante es la opción más adecuada en el sentido de que ataja con éxito la violencia etnocéntrica de la traducción (Venuti, 1992: I3; I995a: 20; Chesterman, I997: 28; Gupta, I998: I86). Sin embargo, la principal diferencia que encuentra Chesterman entre Schleiermacher y Venuti es que, mientras que el primero pretende potenciar la LM, el segundo persigue conseguir mayor reconocimiento para el papel de la LO. El método extranjerizante de la traducción

\footnotetext{
15 «implica elegir un To y poner en práctica un método de traducción basado en aspectos que no aparecen en los valores culturales dominantes de la LM».

16 «negociar estos valores [los diseños discursivos, genéricos y textuales] en términos de las normas y convenciones de la lengua término».

17 «el traductor interviene en el autor lo menos posible $\mathrm{y}$ acerca el lector al autor».
}

es, en palabras de Munday, un estilo de traducción de escasa fluidez diseñado para hacer ver la figura del traductor mediante el énfasis de la identidad extranjera del to y la protección de dicha entidad del predominio ideológico de la cultura meta.

Aunque Venuti se muestra partidario de la traducción extranjerizante, también es consciente de sus contradicciones, como, por ejemplo, el hecho de que se trate de un término subjetivo y relativo en el sentido de que traduce un To para una cultura meta pero al mismo tiempo depende de los valores dominantes de dicha cultura, lo que supone, en definitiva, cierta domesticación (Venuti, I995a: 29; Munday, 200I: I48). A pesar de todo, defiende Venuti el recurso a la traducción extranjerizante, ya que, según su opinión, «Foreignizing translations [...] are equally partial [as are domesticating translations] in their interpretation of the foreign text, but they do tend to flaunt their partiality instead of concealing it ${ }^{18}$ (Venuti, I995a: 34).

Si bien Venuti no ofrece una metodología específica aplicable al análisis de traducciones, recogen Lambert y Van Gorp (I985: 52-53) y Munday (2001: 156) una serie de premisas generales que permiten determinar qué estrategia, de las dos a las que se refiere Venuti, ha prevalecido a la hora de traducir el To:

I) Comparar lingüísticamente del to y el TM en busca de signos que denoten estrategias domesticadoras y extranjerizantes. Para ello deberá observarse la traducción de topónimos, antropónimos y elementos culturales, la división del texto y el empleo de elementos ortotipográficos.

2) Entrevistar al traductor, siempre que sea posible, para averiguar a qué tipo de estrategia

I8 "Las traducciones extranjerizantes [...] son tan parciales como las traducciones domesticadas en lo que a la interpretación del To se refiere, pero tienden a hacer alarde de su parcialidad en vez de disimularla».

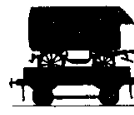




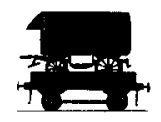

ha recurrido. Analizar las distintas versiones de la traducción si se dispone de ellas.

3) Entrevistar al editor y al agente con obje68

to de conocer sus propósitos de las traducciones, qué criterios utilizan para decidir qué libros se traducen y qué instrucciones les dan al traductor.

4) Averiguar el número de libros traducidos y vendidos, qué libros se eligen, hacia qué lenguas se traducen y cómo evolucionan las tendencias.

5) Estudiar el tipo de contrato firmado para la traducción, así como si el traductor aparece como elemento "visible» en el producto final.

6) Ver si el traductor está literalmente «visible» mediante la comprobación de si el nombre del traductor aparece en la cubierta del libro, a quién se le asignan los derechos de propiedad intelectual, si consta la LO, así como el título del To y, finalmente, si aparece una introducción, dedicatoria o notas al pie en las que quede constancia de que hayan sido realizadas por el traductor.

7) Analizar las reseñas que tengan como objeto la traducción, el autor o el periodo. Con ello se pretende ver qué mención se hace a los traductores y con qué criterio los revisores juzgan la traducción en cuestión.

\section{CONCLUSIONES}

El estudio de la recepción de la literatura traducida, tras unos comienzos un tanto titubeantes, parece haberse consolidado en los últimos años, lo que lo viene a corroborar el que se hayan publicado en los últimos años numerosos estudios que abordan este aspecto desde el punto de vista teórico. $\mathrm{Al}$ mismo tiempo, se advierte un mayor interés en la tercera y más reciente de las ramas de este campo, esto es, la invisibilidad del traductor, hasta el punto de que son abundantes los análisis de obras traducidas que se basan en la opinión de Venuti para estudiar su recepción.
No todo el camino está recorrido, sin embargo, ya que son aún muchos los aspectos que quedan por clarificar. Probablemente el más evidente sea dar con una metodología que permita llegar de forma clara a unas conclusiones homogéneas, puesto que lo que muchos teóricos echan en falta, como hemos recogido a lo largo de este estudio, es una determinación y normalización de los procedimientos que hagan posible fijar si una obra traducida pertenece al centro o a la periferia de un polisistema, conocer cuáles son las normas de traducción que se han empleado y averiguar si el traductor ha optado por la domesticación o la extranjerización como estrategia de traducción.

RECIBIDO ENERO DE 2003

\section{REFERENCLAS BIBLIOGRÁFICAS}

Acosta Gómez, L. A. (1989). El lector y la obra: teoría de la recepción literaria. Madrid: Gredos.

Baker, M. (1998). "Norms», en BAKER, M. (ed.). Routledge Encyclopedia of Translation Studies. Londres; Nueva York: Routledge, pp. I63-165.

- (2000). «Towards a Methodology for Investigating the Style of a Literary Translator", en Target, $12(2)$, pp. $24 I^{-2} 266$.

Bark, K. (r987). «El redescubrimiento del lector: ¿La «estética de la recepción» como superación del estudio inmanente de la literatura?», en RALL, D. (ed.). En busca del texto: teoría de la recepción literaria. México DF: Universidad Nacional Autónoma de México, pp. I7I-I83.

Bassnett, S. (1998). «The Translation Turn in Cultural Studies", en BAssnetT, S.; LeFEvere, A. (ed.). Constructing Cultural Studies: Essays on Literary Translation. Clevedon; Filadelfia; Toronto; Sidney; Johannesburgo: Multilingual Matters, pp. I23-I4O.

Berman, A. (2000). «Translation and the Trials of the Foreign", en VenuTi, L. (ed.). The Translation Studies Reader. Londres; Nueva York: Routledge, pp. 284-297.

Bernofsky, S. (I997). «Schleiermacher's Translation Theory and Varieties of Foreignization: August 
Wilhem Schlegel vs. Johann Heinrich Voss", en The Translator, 3(2), pp. I75-I92.

Chesterman, A. (1997). Memes of Translation: The Spread of Ideas in Translation Theory. Amsterdam; Filadelfia: John Benjamins.

Even-Zohar, I. (1979). «Polysystem Theory», en Poetics Today, I(I-2), pp. 287-310.

- (1981). «Translation Theory Today: A Call for Transfer Theory», en Poetics Today, 2(4), Pp. I-7.

- (1990). "The Position of Translated Literature within the Literary Polysystem", en Poetics Today, II(I), pp. 45-5I.

- (1997). «The Making of Culture Repertoire and the Role of Transferw, en Target, 9(2), pp. 355-363.

Gentzler, E. (1993). Contemporary Translation Theories. Londres; Nueva York: Routledge.

Gupta, P. (1998). «Post- or Neo-Colonial Translation? Linguistic Inequality and Translator's Resistance», en Translation \& Literature, 7(2), pp. I70-193.

Hale, T. (1998). «Publishing Strategies», en BAKER, M. (ed.). Routledge Encyclopedia of Translation Studies. Londres; Nueva York: Routledge, pp. I90-194.

Hatim, B. (I999). "Implications of Research into Translator Invisibility", en Target II(2), pp. 2OI222.

Hermans, T. (1985). «Introduction: Translation Studies and a New Paradigm", en Hermans, T. (ed.). The Manipulation of Literature: Studies in Literary Translation. Londres; Sidney: Croom Helm, pp. 7-I5.

- (r996). "The Translator's Voice in Translated Literature», en Target, 8(I), pp. $23^{-48}$.

- (1999). Translation in Systems: Descriptive and System-oriented Approaches Explained, Translation Theories Explained. Manchester: St. Jerome.

Holmes, J. S. (2000). «The Name and Nature of Translation Studies", en Venuti, L. (ed.). The Translation Studies Reader. Londres; Nueva York: Routledge, pp. 172-185.

Holub, R. C. (1985). Reception Theory: A critical Introduction: Londres; Nueva York: Methuen.

House, T. (1998). "Quality of Translation», en BAKER, M. (ed.). Routledge Encyclopedia of Translation Studies. Londres; Nueva York: Routledge, pp. 212-216.
Iglesias Santos, M. (1994a). «La estética de la recepción y el horizonte de expectativas", en VILLANUEva, D. (comp.). Avances en teoría de la literatura (Estética de la recepción, pragmática, teoria empírica y teoría de los polisistemas). Santiago de Compostela: Universidad, pp. 35-115.

- (r994b). «El sistema literario: teoría empírica y teoría de los polisistemas", en VILlanueVA, D. (comp.). Avances en teoria de la literatura (Estética de la recepción, pragmática, teoría empirica y teoría de los polisistemas). Santiago de Compostela: Universidad, pp. 309-356.

Iser, W. (1987a). «El acto de la lectura: consideraciones previas sobre una teoría del efecto estético", en RALL, D. (ed.). En busca del texto: teoría de la recepción literaria. México DF: Universidad Nacional Autónoma de México, pp. 121-143.

- (1987b). «A la luz de la crítica», en RALL, D. (ed.). En busca del texto: teoría de la recepción literaria. México DF: Universidad Nacional Autónoma de México, pp. $145^{-160}$

Jauss, H. R. (I987a). «Cambio de paradigma en la ciencia literaria», en RALL, D. (ed.). En busca del texto: teoría de la recepción literaria. México DF: Universidad Nacional Autónoma de México, pp. 59-7I.

- (1987b). «Experiencia estética y hermenéutica literaria», en RALL, D. (ed.). En busca del texto: teoria de la recepción literaria. México DF: Universidad Nacional Autónoma de México, pp. 73-87.

Johnston, J. (1992). «Translation as Simulacrum», en Venuti, L. (ed.). Rethinking Translation. Discourse, Subjectivity, Ideology. Londres; Nueva York: Routledge, pp. 42-56.

Jurt, J. (I998). «Les-arten. Rezeptions- und Lektüreforschung und ihre Folgen für das Literaturverständnis», en Revista de Filologia Alemana, 6, pp. $43-68$.

Lambert, J. (I998). «Literary Translation: Research Issuesm, en BAKER, M. (ed.). Routledge Encyclopedia of Translation Studies. Londres; Nueva York: Routledge, pp. 130-133.

Lambert, J.; Van Gorp, H. (1985). «On Describing Translation", en Hermans, T. (ed.). The Manipulation of Literature: Studies in Literary Translation. Londres; Sidney: Croom Helm, pp. 42-53.

Lefevere, A. (1995). «Introduction: Comparative Literature and Translation», en Comparative Literature, $47(\mathrm{I})$, Pp. I-IO.

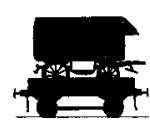

69 


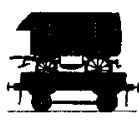

Lefevere, A.; Bassnett, S. (1998). «Introduction: Where are we in Translation Studies?», en BassNeT, S.; Lefevere, A. Constructiong Cultures: Essays on Literary Translation. Clevedon; Filadelfia; Toronto; Sidney; Johannesburgo: Multilingual Matters, pp. I-II.

Marco, J. (2002). El fil d'Ariadna. Anàlisi estilistica i traducció literària, Biblioteca de Traducció i Interpretació. Vic: Eumo.

Munday, J. (200I). Introducing Translations Studies: Theories and Applications. Londres; Nueva York: Routledge.

Pym, A. (1996). «Review Article: Venuti's Visibilitys, en Target, 8(r), pp. 165-177.

Schäffner, C. (1995). "Editorial», en SCHAFFNER, C.; Kelly-Holmes, H. (eds.). Cultural Functions of Translation. Clevedon; Filadelfia: Adelaide, pp. I-8.

Segers, R. T. (1979). "An Interview with Hans Robert Jaus», en New Literary History, rr(I), pp. 83-95.

Shuttleworth, M. (1998). "Polysystem Theory», en BAKER, M. (ed.). Routledge Encyclopedia of Translation Studies. Londres; Nueva York: Routledge, pp. I76-r79.

Starobinski, J. (r987). "Un desafio a la teoría literariam, en RaLl, D. (ed.). En busca del texto: teoría de la recepción literaria. México DF: Universidad Nacional Autónoma de México, pp. 2rr-220.

Toury, G. (1980). In Search of a Theory of Translation. Tel Aviv: The Porter Institute for Poetics and Semiotics.

- (r98I). «Translated Literature: System, Norm, Performance: Toward a TT-Oriented Approach to Literary Translation», en Poetics Today, 2(4), pp. $9^{-27}$.
- (r985). "A Rationale for Descriptive Translation", en Hermans, T. (ed.). The Manipulation of Literature: Studies in Literary Translation. Londres; Sidney: Croom Helm, pp. 16-4I.

- (2000). "The Nature and the Role of Norms in Translation». VENUTI, L. (ed.). The Translation Studies Reader. Londres; Nueva York: Routledge, pp. I98-2II.

Vanderschelden, I. (2000). «Quality Assessment and Literary Translation in Francen, en The Translator, 6(2), pp. 27r-293.

Venuti, L. (I985). «The Ideology of the Individual in Anglo-American Criticism: The Example of Coleridge and Eliot", en Boundary 2, $14(\mathrm{I}-2)$, pp. I6I-I93.

- (I992). «Introduction», en VENUTI, L. (ed.). Retbinking Translation. Discourse, Subjectivity, Ideology. Londres; Nueva York: Routledge, pp. I-I7.

- (r995a). The Translator's Invisibility: A History of Translation. Londres; Nueva York: Routledge.

- (r995b). «Translation and the Formation of Cultural Identities», en SChäffner, C.; KellyHolmes, H. (eds.). Cultural Functions of Translation. Clevedon; Filadelfia: Adelaide, pp. 9-25.

- (I995c). «Preliminary Remarks to the Debatem, en Schäffner, C.; Kelly-Holmes, H. (eds.). Cultural Functions of Translation. Clevedon; Filadelfia: Adelaide, pp. 26-31.

- (1998a). «The American Tradition», en BAKER, M. (ed.). Routledge Encyclopedia of Translation Studies. Londres; Nueva York: Routledge, pp. 305-315.

- (r998b). The Scandals of Translation: Towards an Ethics of Difference. Londres; Nueva York: Routledge.

Weinrich, H. (1987). «Para una historia literaria del lector", en RaLL, D. (ed.). En busca del texto: teoría de la recepción literaria. México $\mathrm{DF}$ : Universidad Nacional Autónoma de México, pp. 199-210. 